\title{
USO DE CAL HIDRATADA AGRÍCOLA EM SISTEMA DE SEMEADURA DIRETA DE SOJA NO NOROESTE PAULISTA
}

\author{
BORGES, Wander Luis Barbosa ${ }^{1}$; HIPÓLITO, Jorge Luiz ${ }^{2}$; SOUZA, Isabela Malaquias Dalto de ${ }^{3}$; \\ ANDREOTTI, Marcelo ${ }^{4}$; TOKUDA, Flávio Sueo ${ }^{5}$ GOMES, Edvaldo Novelli ${ }^{6}$; STRADA, Wilson \\ Luiz $^{5}$
}

RESUMO: Há pouca informação sobre a utilização da cal hidratada agrícola em sistemas sustentáveis de produção agropecuária, principalmente no cultivo de soja em sistema de semeadura direta. O presente trabalho foi realizado com o objetivo avaliar o efeito do uso de cal hidratada agrícola sobre a cultura da soja cultivada no sistema de semeadura direta na região Noroeste Paulista. O delineamento experimental utilizado foi em blocos casualizados com quatro tratamentos (T1: tratamento padrão (sem aplicação de cal hidratada agrícola); T2 - aplicação de cal hidratada agrícola em superfície para estabelecer que o cálcio ocupe 70\% da capacidade de troca de cátions (CTC) na camada de 0-0,20 m; T3 - aplicação de cal hidratada agrícola em superfície para estabelecer que o cálcio ocupe $52,5 \%$ da CTC na camada de 0-0,20 m; T4 - aplicação de cal hidratada agrícola em superfície para estabelecer que o cálcio ocupe $35 \%$ da CTC na camada de $0-0,20 \mathrm{~m}$.) e quatro repetições. Os parâmetros avaliados na cultura da soja foram: altura de inserção da primeira vagem, altura de plantas, estande final $\mathrm{ha}^{-1}$, massa de cem grãos e produtividade de grãos ha ${ }^{-1}$. Os dados foram submetidos ao teste $\mathrm{F}$ e as médias foram comparadas pelo teste de Tukey $(\mathrm{p}<0,05)$. Constatou-se que o tratamento T2 apresentou maior altura de inserção da primeira vagem que o tratamento padrão. Para as demais características agronômicas avaliadas não houve diferença entre os tratamentos.

Palavras-chave: Glycine max (L.) Merrill. Corretivos. Características agronômicas.

\section{AGRICULTURAL HYDRATED LIME USE IN NO-TILLAGE OF SOYBEAN IN THE NORTHWEST REGION OF SÃO PAULO STATE, BRAZIL}

\begin{abstract}
SUMMARY: There is little information on the use of agricultural hydrated lime in sustainable agricultural production systems, mainly in the cultivation of soybean in no-tillage. The present work was carried out with the objective of evaluating the effect of hydrated agricultural lime application on soybean cultivation in no-tillage in the Northwest region of São Paulo State, Brazil. The experimental design was a randomized block with four treatments (T1: standard treatment (without application of agricultural hydrated lime); T2 - application of hydrated agricultural lime in surface to establish that calcium occupies $70 \%$ of the CTC in the $0-0.20 \mathrm{~m}$ layer, T3 - application of hydrated agricultural lime in surface to establish that calcium occupies $52.5 \%$ of the CTC in the $0-0.20 \mathrm{~m}$ layer, T4 application of agricultural hydrated lime in surface to establish that calcium occupies $35 \%$ of CTC in the $0-0.20 \mathrm{~m}$ layer) and four replicates. The parameters evaluated in the soybean crop were: height of insertion of the first pod, height of plants, final stand $\mathrm{ha}^{-1}$, mass of one hundred grains and grain productivity ha ${ }^{-1}$. The data were submitted to the $\mathrm{F}$ test and the means were compared by the Tukey test $(\mathrm{p}<0.05)$. It was verified that the treatment $\mathrm{T} 2$ presented higher height of insertion of the first pod than the standard treatment. For the other evaluated agronomic characteristics, there was no difference between treatments.
\end{abstract}

Keywords: Glycine max (L.) Merrill. Correctives. Agronomic characteristics.

\footnotetext{
${ }^{1}$ Pesquisador Científico, Dr. - IAC - CAP de Seringueira e Sistemas Agroflorestais, Votuporanga, SP;

${ }^{2}$ Assistente Agropecuário - CDRS - DSMM/NPS, Araçatuba, SP;

${ }^{3}$ Doutoranda, MSc. - UNESP - Faculdade de Engenharia, Ilha Solteira, SP. Bolsista FAPESP;

${ }^{4}$ Professor Adjunto, Dr. - UNESP - Faculdade de Engenharia, Ilha Solteira, SP.

${ }^{5}$ Assistente Agropecuário - CDRS - DSMM/NPS, Fernandópolis, SP;

${ }^{6}$ Técnico de apoio - IAC - CAP de Seringueira e Sistemas Agroflorestais, Votuporanga, SP.
} 


\section{INTRODUÇÃO}

A adoção de sistemas sustentáveis de produção agropecuária é fundamental para o contínuo avanço tecnológico da agricultura brasileira. Entre esses sistemas está o sistema de semeadura direta, que exclui as práticas de revolvimento do solo, permitindo o acúmulo de material vegetal na superfície, sobre o qual será semeada ou plantada a cultura seguinte (BERTIN et al., 2005).

Neste sistema, geralmente, a correção da acidez do solo é realizada mediante aplicação de calcário na superfície, sem incorporação e, a baixa mobilidade dos produtos de dissolução do calcário aplicado na superfície limita sua eficiência na redução da acidez em camadas sub superficiais de solos com cargas variáveis e, que dependem da lixiviação de sais, orgânicos e, ou, inorgânicos, através do perfil do solo (CAIRES et al., 2006).

Uma alternativa para se ajustar o $\mathrm{pH}$ dos solos e proporcionar melhores condições de crescimento radicular e, assim, melhorar o rendimento das culturas, é a utilização de carbonato de cálcio, cal virgem e cal hidratada. A cal tem um efeito benéfico no solo de neutralizar os ácidos prejudiciais e aumentar a humificação da matéria orgânica, tornando o solo mais fértil. Tanto o carbonato de cálcio como a cal virgem são usados como intensificadores da qualidade do solo (EULA, 2018).

Há pouca informação sobre a utilização da cal hidratada agrícola em sistema de semeadura direta, principalmente no cultivo de soja, assim, o presente trabalho foi realizado com o objetivo de avaliar o efeito da aplicação de cal hidratada agrícola sobre a cultura da soja cultivada no sistema de semeadura direta na região Noroeste Paulista.

\section{MATERIAL E MÉTODO}

O experimento foi instalado no Centro Avançado de Pesquisa de Seringueira e Sistemas Agroflorestais, do Instituto Agronômico (IAC), da Agência Paulista de Tecnologia dos Agronegócios APTA/SAA, localizado no município de Votuporanga, SP, (20²0'S, $49^{\circ} 58^{\prime} \mathrm{W}$ e $510 \mathrm{~m}$ de altitude), em um Argissolo Vermelho-Amarelo eutrófico (segundo o SiBCS, SANTOS et al., 2013).

O clima é o tropical com invernos secos (Aw na classificação de Köppen) com temperatura média anual de $24^{\circ} \mathrm{C}$, tendo a média das máximas de $31,2^{\circ} \mathrm{C}$ e a média das mínimas de $17,4^{\circ} \mathrm{C}$, enquanto que a precipitação pluvial média anual é de $1328,6 \mathrm{~mm}$.

A área do experimento era destinada à produção de grãos, com sistema convencional de preparo do solo. Na safra 2008/09 a área foi cultivada com amendoim e sorgo granífero sobre a resteva do amendoim. Após o cultivo do sorgo, todas as semeaduras foram realizadas em sistema de semeadura direta. O sistema de rotação de culturas adotado no sistema, no período de novembro de 2009 a outubro de 2018, consta na Tabela 1. O sorgo forrageiro, a Urochloa ruziziensis e a U. brizantha foram utilizados como plantas de cobertura e não foram retirados da área. A quantidade de nutrientes utilizada no sistema, no período de novembro de 2009 a outubro de 2018, encontra-se na Tabela 2. 
Tabela 1. Rotação de cultura utilizada no período de novembro (Nov) de 2009 a outubro (Out) de 2018.

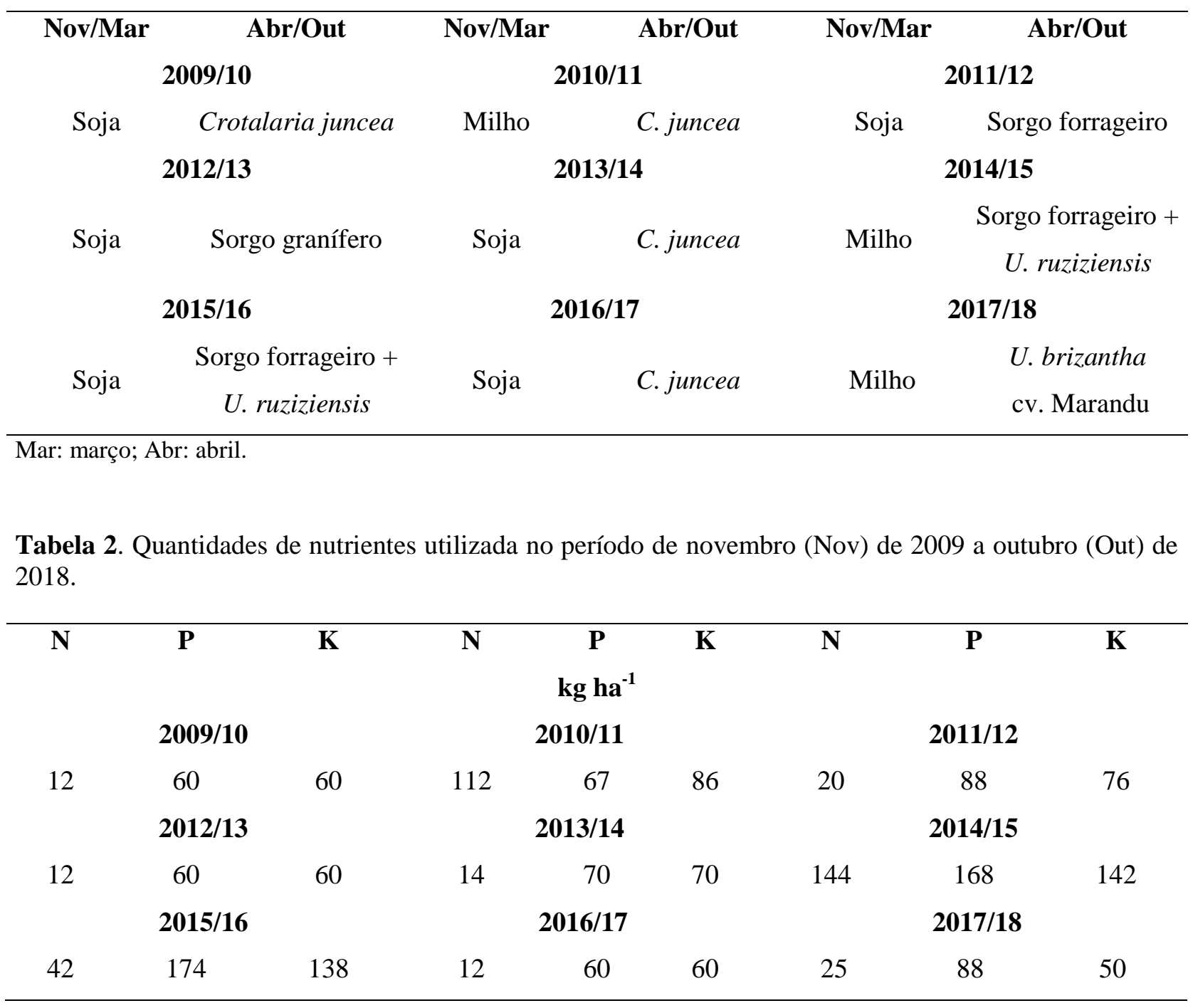

Foi realizada uma coleta de solo no dia 16/04/2018 para análise química de solo e determinação da sua fertilidade (RAIJ et al., 2001), nas camadas de 0-0,20 e 0,20-0,40 m de profundidade, e os resultados estão apresentados na Tabela 3.

Tabela 3. Valores referentes à análise de amostra de solo, em diferentes sistemas de produção, nas camadas de 0-0,20 e 0,20-0,40 m, 2018.

\begin{tabular}{|c|c|c|c|c|c|c|c|c|c|c|}
\hline \multirow{2}{*}{ Camadas } & $\mathbf{P}$ & ${\mathrm{S}-\mathrm{SO}_{4}}$ & MO & pH & $\mathbf{K}$ & $\mathbf{C a}$ & Mg & $\mathbf{H}+\mathbf{A l}$ & Al & $\mathbf{V}$ \\
\hline & \multicolumn{2}{|c|}{$\mathbf{m g ~ d m} \mathbf{m}^{-3}$} & \multicolumn{2}{|c|}{$\mathrm{g} \mathrm{dm}^{-3}$} & \multicolumn{5}{|c|}{ 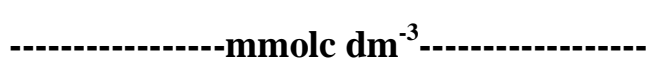 } & $\%$ \\
\hline $0-0,20$ & 25 & 4 & 15 & 5,1 & 1,3 & 17 & 14 & 26 & 1 & 53 \\
\hline $0,20-0,40$ & 17 & 5 & 13 & 4,0 & 1,8 & 8 & 6 & 34 & 6 & 33 \\
\hline
\end{tabular}

O delineamento experimental utilizado foi o em blocos casualizados com quatro repetições, utilizando-se quatro tratamentos: T1 - tratamento padrão (sem aplicação de cal hidratada agrícola); T2 aplicação de cal hidratada agrícola em superfície para estabelecer que o cálcio ocupe $70 \%$ da capacidade de troca de cátions (CTC) na camada de 0-0,20 m; T3 - aplicação de cal hidratada agrícola em superfície 
para estabelecer que o cálcio ocupe 52,5\% da CTC na camada de 0-0,20 m; T4 - aplicação de cal hidratada agrícola em superfície para estabelecer que o cálcio ocupe 35\% da CTC na camada de 0-0,20 m.

Cada parcela foi composta por dez linhas de soja (espaçamento de $0,50 \mathrm{~m}$ ) e $5 \mathrm{~m}$ de comprimento, totalizando $25 \mathrm{~m}^{2}$.

A cal hidratada agrícola foi aplicada manualmente sobre a superfície do solo, no dia 30/08/2018.

No dia 27/11/2018 foi realizada uma roçada da $U$. brizantha presente na área para facilitar a semeadura da soja, pois a mesma apresentava, em média, $18840 \mathrm{~kg} \mathrm{ha}^{-1}$ de matéria verde.

A semeadura da soja foi realizada mecanicamente no sistema de semeadura direta sobre a palhada da $U$. brizantha no dia 24/11/2017, utilizando a cultivar 74HO112TP IPRO Paranaiba no espaçamento de $0,50 \mathrm{~m}$ e população de 280000 plantas ha ${ }^{-1}$, com adubação de base na dose de $300 \mathrm{~kg} \mathrm{ha}^{-1}$ do formulado 04-20-20.

Os parâmetros avaliados na cultura da soja foram: altura de inserção da primeira vagem, altura de plantas, estande final ha ${ }^{-1}$, massa de cem grãos e produtividade de grãos $\mathrm{ha}^{-1}$.

As avaliações foram realizadas no momento da colheita da cultura da soja, realizada no dia 01/04/2019. A massa de cem grãos e produtividade de grãos foi obtida padronizando-se a umidade dos grãos para $13 \%$ (base úmida).

A amostragem da altura de inserção da primeira vagem e altura de plantas foi realizada em cinco plantas de cada parcela, e a amostragem do estande final ha ${ }^{-1}$, massa de cem grãos e produtividade de grãos foi realizada em $3 \mathrm{~m}$ de duas linhas centrais de cada parcela.

As vagens foram debulhadas em debulhadora mecânica. Após a debulha os grãos foram pesados e mensurada sua umidade para o cálculo da produtividade de grãos. Em seguida separou-se cem grãos para cálculo da massa de cem grãos.

Os dados foram submetidos ao teste $\mathrm{F}$ e as médias foram comparadas pelo teste de Tukey ( $\mathrm{p}<0,05)$, com o uso do programa computacional Assistat (SILVA; AZEVEDO, 2016).

Os dados mensais de evapotranspiração potencial, precipitação pluvial e temperatura média de Votuporanga, SP, no período de novembro de 2018 a abril de 2019, encontram-se na Figura 1.

Figura 1. Dados de evapotranspiração potencial (ETP), precipitação pluvial (PP) e temperatura média (T), em Votuporanga, SP, no período estudado, novembro de 2018 a abril de 2019. Fonte: CIIAGRO (2019).

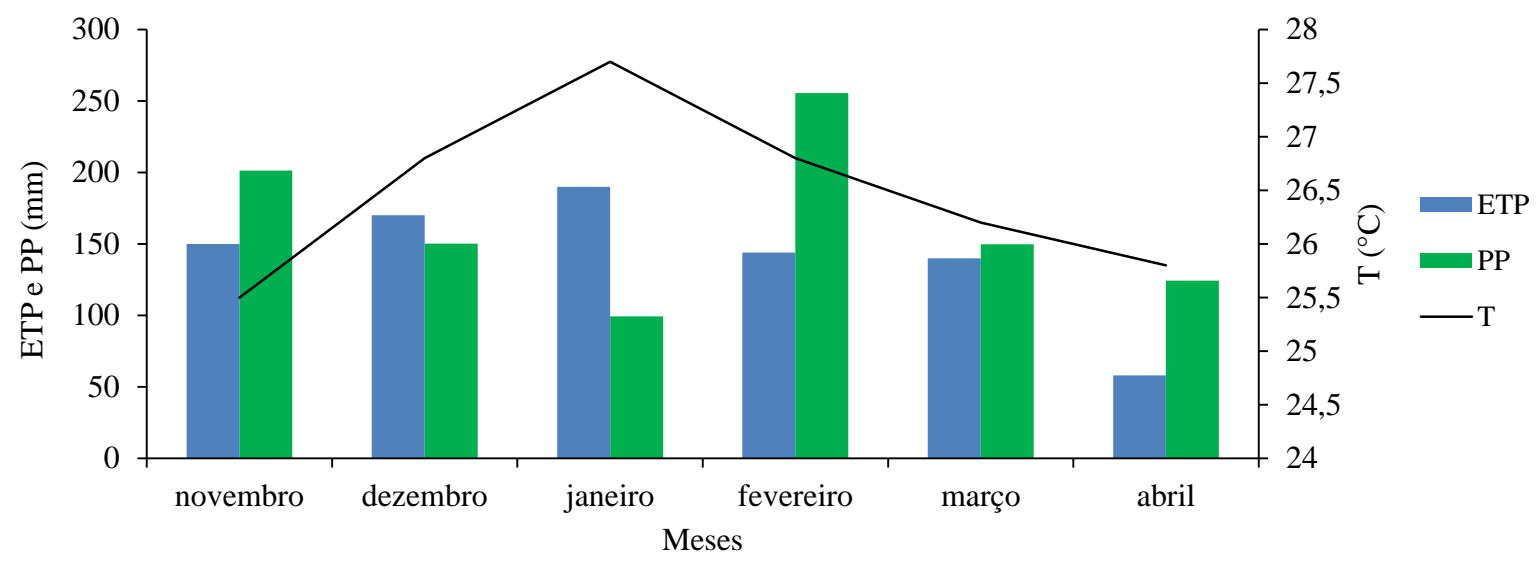

\section{RESULTADO E DISCUSSÃO}

As características agronômicas da cultura da soja estão demonstradas na Tabela 4. O tratamento T2 (aplicação de cal hidratada agrícola em superfície para estabelecer que o cálcio ocupe 70\% da CTC na 
camada de $0-0,20 \mathrm{~m})$ apresentou maior altura de inserção da primeira vagem e diferiu $(\mathrm{p}<0,05)$ do tratamento padrão (sem aplicação de cal hidratada agrícola), no entanto, todos os tratamentos apresentaram altura de inserção inferior a 0,12 m. Segundo Yokomizo (1999), valores de inserção da primeira vagem inferiores a $0,12 \mathrm{~m}$ podem resultar em perdas na colheita e, em consequência, reduzir os ganhos dos produtores.

Todas as cultivares apresentaram altura de plantas superior a $0,65 \mathrm{~m}$. De acordo com Bonetti (1983), a altura mínima de plantas recomendada para colheita mecânica é de 0,65 m, considerando-se que plantas de menor altura tendem a produzir vagens igualmente baixas e, portanto, difíceis de serem colhidas mecanicamente (LAZARINI, 1995).

Para as características altura de plantas, estande final ha ${ }^{-1}$, massa de cem grãos e produtividade de grãos ha ${ }^{-1}$ não houve diferença $(\mathrm{p}<0,05)$ entre os tratamentos.

Tabela 4. Características agronômicas da cultura da soja, Votuporanga, SP, 2019.

\begin{tabular}{lcccccc}
\hline Tratamentos $^{(\mathbf{1})}$ & $\begin{array}{c}\text { Altura de } \\
\text { inserção }^{(\mathbf{4})}\end{array}$ & $\begin{array}{c}\text { Altura de } \\
\text { plantas }\end{array}$ & $\begin{array}{c}\text { Estande final } \\
(\mathbf{m})\end{array}$ & $\begin{array}{c}\text { Massa de cem } \\
\text { grãos }\end{array}$ & $\begin{array}{c}\text { Produtividade } \\
\text { de grãos }\end{array}$ \\
T1 - Padrão & 0,04 & $\mathbf{b}^{(5)}$ & $0,78^{(\mathrm{ns})}$ & 139167 & 14,27 & 4735 \\
T2 & 0,07 & $\mathrm{a}$ & 0,80 & 133333 & 15,16 & 5043 \\
$\mathrm{~T} 3$ & 0,05 & $\mathrm{ab}$ & 0,78 & 133333 & 14,50 & 4639 \\
$\mathrm{~T} 4$ & 0,05 & $\mathrm{ab}$ & 0,90 & 131667 & 14,91 & 4818 \\
$\mathrm{DMS}^{(2)}$ & 0,03 & 0,14 & 55430 & 1,53 & 1775 \\
$\mathrm{CV}^{(3)}$ & 22,49 & 7,77 & 18,67 & 4,70 & 16,70 \\
\hline
\end{tabular}

(1) T1: 70\% da CTC (0-0,20 m); T2: 52,5\% da CTC (0-0,20 m); T3: 35\% da CTC (0-0,20 m); ${ }^{(2)}$ DMS: Diferença mínima significativa; ${ }^{(3)} \mathrm{CV}$ : Coeficiente de variação; ${ }^{(4)}$ Altura de inserção: altura de inserção da primeira vagem; ${ }^{(5)}$ significativo a $5 \%$ de probabilidade; ${ }^{\text {(ns) }}$ : não-significativo.

\section{CONCLUSÃO}

A aplicação de cal hidratada agrícola em superfície, para estabelecer que o cálcio ocupe $70 \%$ da CTC na camada de 0-0,20 m, proporciona maior altura de inserção da primeira vagem da cultura da soja, em sistema de semeadura direta, no Noroeste Paulista.

\section{AGRADECIMENTOS}

A todos os funcionários do Centro Avançado de Pesquisa de Seringueira e Sistemas Agroflorestais, do Instituto Agronômico - IAC, pelo apoio na instalação e condução do experimento.

\section{REFERÊNCIAS}

BERTIN, E. G.; ANDRIOLI, I.; CENTURION, J. F. Plantas de cobertura em pré-safra ao milho em plantio direto. Acta Scientiarum. Agronomy, Maringá, v. 27, n. 3, p. 379-386, 2005. Disponível em: http://dx.doi.org/10.4025/actasciagron.v27i3.1393 
BONETTI, L. P. Cultivares e seu melhoramento genético. In: VERNETTI, F. J. (Coord.) Soja: genética e melhoramento. Campinas: Fundação Cargill, 1983. p. 741-94.

CAIRES, E. F.; BARTH, G.; GARBUIO, F. J. Lime application in the establishment of a no-till system for grain crop production in Southern Brazil. Soil and Tillage Research, v. 89, n. 1, p. 3-12, 2006. Disponível em: https://doi.org/10.1016/j.still.2005.06.006

CENTRO INTEGRADO DE INFORMAÇÕES AGROMETEOROLÓGICAS - CIIAGRO. Resenha: Votuporanga no período de 01/11/2018 até 30/04/2019. São Paulo, 2019. Disponível em: http://www.ciiagro.sp.gov.br/ciiagroonline/Listagens/Resenha/LResenhaLocal.asp

EUROPEAN LIME ASSOCIATION AISBL - EULA. Lime applications: agriculture, forestry and fish farming. 2018. Disponível em: https://www.eula.eu/agriculture-forestry-and-fish-farming

LAZARINI, E. Avaliação das características agronômicas e análises nutricionais de genótipos de soja semeados em diferentes épocas, em Jaboticabal, SP. 1995. 197 f. Tese (Doutorado em Agronomia Produção Vegetal) - Universidade Estadual Paulista, Jaboticabal.

RAIJ, B. van. et al. Análise química para avaliação da fertilidade do solo. Campinas: Instituto Agronômico; 2001.

SANTOS, H. G.et al. Centro Nacional de Pesquisa de Solos. Sistema brasileiro de classificação de solos. 3. ed. Rio de Janeiro, 2013. 353 p.

SILVA, F. A. S.; AZEVEDO, C. A. V. The Assistat Software Version 7.7 and its use in the analysis of experimental data. African Journal of Agricultural Research, v. 11, n. 39, p. 3733-3740, 2016. Disponível em: http://www.academicjournals.org/journal/AJAR/article-abstract/5E8596460818

YOKOMIZO, G. K. Interação genótipos $\mathbf{x}$ ambientes em topocruzamentos de soja tipo alimento com tipo grão. 1999. 170 f. Tese (Doutorado) - Universidade de São Paulo, Piracicaba, 1999. 